\title{
EchoGéo
}

$11 \mid 2010$

Madagascar

\section{Contraintes et enjeux de développement de la Nouvelle-Calédonie}

Quel schéma d'aménagement et de développement à l'horizon 2025 ?

\section{Christian Jost}

\section{OpenEdition}

\section{Journals}

Édition électronique

URL : https://journals.openedition.org/echogeo/11612

DOI : 10.4000/echogeo.11612

ISSN : 1963-1197

Éditeur

Pôle de recherche pour l'organisation et la diffusion de l'information géographique (CNRS UMR 8586)

\section{Référence électronique}

Christian Jost, «Contraintes et enjeux de développement de la Nouvelle-Calédonie », EchoGéo [En ligne], 11 | 2010, mis en ligne le 24 février 2010, consulté le 31 juillet 2021. URL : http:// journals.openedition.org/echogeo/11612 ; DOI : https://doi.org/10.4000/echogeo.11612

Ce document a été généré automatiquement le 31 juillet 2021.

EchoGéo est mis à disposition selon les termes de la licence Creative Commons Attribution - Pas d'Utilisation Commerciale - Pas de Modification 4.0 International (CC BY-NC-ND) 


\title{
Contraintes et enjeux de développement de la Nouvelle- Calédonie
}

Quel schéma d'aménagement et de développement à l'horizon 2025 ?

\author{
Christian Jost
}

L'auteur remercie tout particulièrement Madame Muriel Texier-Dos Santos, Coordinatrice du Service de l'aménagement et de la planification du Gouvernement de la Nouvelle-Calédonie, pour l'accès aux documents du SADNC et pour l'aide apportée à la réalisation de cet article.

1 Avec le lancement officiel de la démarche « Nouvelle-Calédonie 2025 » en mai 2008, la Nouvelle-Calédonie a ouvert un nouveau chapitre de son histoire contemporaine et non des moindres, puisqu'il s'agit de préparer le schéma d'aménagement et de développement du territoire à l'horizon 2025. Sous l'égide du gouvernement local et de l'Etat français, la première conférence des acteurs qui s'est tenue le 14 mai 2008 à Koné, en Province Nord, a initié une vaste consultation destinée à préparer le "Schéma d'Aménagement et de Développement de la Nouvelle-Calédonie " (SADNC ou SAD). Ce schéma a pour objectif de permettre aux politiques publiques d'être plus efficaces et plus en adéquation avec les réalités d'aujourd'hui, en adaptant les réponses à apporter aux contraintes et aux enjeux de société et de "pays ». Les signes avant-coureurs d'une crise financière mondiale ressentis en 2007 ont-ils précipité la mise en place de ces Etats-généraux sur l'avenir de la Nouvelle-Calédonie? Rien n'empêche de le penser, même si ceux-ci étaient prévus de longue date.

2 En effet, faisant suite à l'Accord de Nouméa signé en 1998, la loi organique n99-209 du19 mars 1999 prévoit, dans son article 211, la mise en place d'un tel schéma qui « exprime les orientations fondamentales en matière d'infrastructures, de formation initiale et continue, d'environnement, d'équipements, de services d'intérêt territorial et de développement économique, social et culturel » et qui « veille à un développement équilibré du territoire ». Le titre VIII, qui contient cet article, envisage «le rééquilibrage et le développement économique, social et culturel» au travers, dans un premier temps, de contrats pluriannuels de développement conclus sur cinq ans entre l'Etat d'une part, la 
Nouvelle-Calédonie et les Provinces d'autre part et, dans un deuxième temps au travers d'un schéma de développement. Une première tentative d'élaboration du SADNC fut menée durant l'année 2000. Neuf groupes de travail correspondant aux thématiques de l'article 211 produisirent Etat des lieux, Etat des besoins et Objectifs. Une première synthèse de l'état des lieux fut réalisée à partir de ces travaux mais resta lettre morte. Le projet fut relancé en 2001 et permit d'aboutir en mai 2002 à une version plus complète, actualisée et cartographiée de l'Etat des lieux qui fut approuvé par le comité de pilotage du SAD. Cependant, en l'absence de volonté politique et de moyens humains dévolus à ce projet, il fallut attendre 2004 pour que le processus soit relancé avec Gérald Cortot, en charge du SADNC, et par la création en juin 2006 d'un Service de l'aménagement et de la planification. Ces nouveaux éléments et l'engagement cette fois de tous les acteurs institutionnels ont permis de jeter les bases d'une consultation participative pour réfléchir sur la question de fond : «Quels contours donner au pays à l'horizon 2025 ?».

3 Les défis à relever et les contraintes à surmonter sont multiples dans ce territoire multiculturel et pluriethnique, au passé commun douloureux et au présent encore difficile pour les différentes communautés qui cohabitent plus qu'elles n'échangent réellement.

En effet, le plus grand défi que doit relever la population (nous préférons dire «les populations ») de Nouvelle-Calédonie, réside dans la recherche, la « cristallisation » et l'appropriation "d'une identité calédonienne", si tant est que l'idée d'une seule identité soit la meilleure voie pour un projet d'avenir. Le sujet est aussi d'actualité en métropole et semble bien préoccuper les dirigeants français qui lancent le débat sur «l'identité nationale ». Certaines voix s'élèvent et font justement état de la richesse de la diversité et d'une identité française, plus que nationale, qui se nourrit de multiples racines.

5 Par le biais d'un grand projet commun d'avenir, comme le SAD, peut certes être esquissée une communauté de destin. De là à forger une "identité calédonienne» commune, il y a un pas qu'il nous apparaît difficile de franchir dans le court terme, tant les divergences de vues sur la politique de pays à mener apparaissent encore importantes. Les dirigeants locaux doivent en effet intégrer et composer avec des liens, des enjeux et des intérêts qui sont souvent en conflit (montée en puissance du secteur nickel et protection d'un environnement exceptionnel, revendications de terres et concessions minières, terres coutumières et croissance urbaine, sociétés familiales et pouvoir local de familles, etc.) dans un cadre législatif particulier à la fois de droit commun et de droit local. Ils doivent aussi répondre aux exigences et pressions économiques, extérieures et intérieures, ainsi qu'aux demandes et aspirations sociales des différentes communautés. Ceci suppose une véritable et sincère mise en plat, un état des lieux réalisé par tous les acteurs du développement et permettant à la population d'y participer, de s'exprimer. C'est en ce sens qu'a été lancée en mai 2008 la première conférence des acteurs du développement. L'auteur de cet article n'y a pas participé, mais a été sollicité comme «Grand Témoin » au terme du processus, en 2009, pour une analyse du bilan de cette consultation. N'ayant pu y répondre dans les délais impartis, c'est à travers cet article que sont analysés à la fois les contraintes et enjeux de développement du territoire et le bilan de cette consultation qui posent les questions suivantes : 
6 Quel est le contexte actuel de la Nouvelle-Calédonie dans lequel ce diagnostic a été réalisé? Quelles contraintes et quels enjeux de développement doivent retenir et/ou retiennent déjà les Calédoniens? Quels sont les résultats de la consultation à ce jour ? $\mathrm{Au}$-delà de cette consultation, quelles réponses et quelles pistes peuvent être esquissées? Et quel bilan peut-on en faire?

\section{Contexte économique et politique}

7 Tant du point de vue économique, politique que culturel, le monde calédonien est un monde biface, partagé entre deux grandes visions du Monde, entre deux acceptions et acceptations du concept, importé, de "développement». L'une est occidentale et fonctionne selon les règles et les valeurs du capitalisme et de l'individualisme, l'autre est océanienne et est régie par des règles et des valeurs communautaires. Rappelons que, même sur le plan juridique, coexistent le droit républicain et un droit local coutumier reconnu aux Kanak. Il n'est cependant pas bipolaire au sens économique, même si les tentatives de rééquilibrage du territoire, avec le développement du complexe VKP (Voh, Koné, Pouembout) au nord, visent à créer un contre-poids économique et social à la suprématie de l'agglomération du Grand Nouméa.

8 Si l'on s'en tient au mode d'évaluation de la «performance» ou «degré de développement » d'une région ou d'un pays selon les critères économiques employés par les grandes agences, tel que résumé ci-dessous, on s'aperçoit vite que les grilles d'analyse «à l'occidentale » sont essentiellement quantitatives et ne sont pas adaptées pour prendre en compte les modes de fonctionnement locaux, notamment en brousse, qui relèvent pour une large part d'un système d'agriculture de proximité et d'économie que l'OCDE qualifierait d'informelle.

9 Après une période de croissance économique soutenue de 1998 à 2007 (3,7\% en rythme annuel moyen), et forte depuis $2000 \mathrm{du}$ fait de ce l'on peut qualifier de deuxième boom $\mathrm{du}$ nickel, après celui des années 1970, l'économie du territoire est aussi rattrapée depuis 2008 par la crise financière mondiale. Malgré des indicateurs (utilisés par les analystes occidentaux) qui témoignent d'une activité économique encore bonne, la dégradation $\mathrm{du}$ secteur nickel tire la croissance vers le bas. Si on retient provisoirement ces indicateurs en l'absence d'autres données, la croissance réelle de 2008 est ainsi passée à $+0,2 \%$ (contre $+5,6 \%$ en 2007 ) pour l'ensemble de l'économie et à $+4,0 \%$ hors nickel (ISEE, 2008). Dans l'ensemble, le niveau de vie en Nouvelle-Calédonie est aujourd'hui comparable à celui de la grande majorité des régions françaises. Le PIB par habitant est proche de celui de Singapour et supérieur à celui de la Nouvelle-Zélande. Le taux d'investissement est supérieur à $30 \%$, comparable à celui de l'Inde et nettement supérieur à celui de la France métropolitaine (20\%) (CEROM, 2008). Cet investissement est majoritairement le fait des entreprises privées et des ménages.

Mais le coût de la vie reste élevé. L'indexation des salaires des fonctionnaires, calculée sur le coût de la vie, est à Nouméa de 1,73 fois celui de la métropole. L'isolement géographique, l'étroitesse du marché intérieur, la politique de protection tarifaire, les salaires élevés, etc., sont les principaux responsables de la cherté de la vie, rappelle le CEROM $^{1}$ (CEROM, 2008). Il est intéressant et curieux de noter que le CEROM publie des chiffres de coût de la vie basés sur "l'indice BigMac² » utilisé par les économistes de l'OCDE quand les données pour calculer une "parité de pouvoir d'achat" (PPA) sont insuffisantes ; « en considérant cet indicateur, le niveau moyen des prix en Nouvelle-Calédonie 
était, en 2007, de 14\% supérieur à celui de la zone euro, de $45 \%$ à celui de la Nouvelle-Zélande, de $70 \%$ à celui de l'Australie et de 90\% à celui de Singapour " (CEROM, 2008). Même si cet indice peut permettre de grossières comparaisons de pays à pays, ou plutôt de villes à villes où sont installés des « Mc Donald's », son utilisation en Océanie insulaire et en particulier en Nouvelle-Calédonie, où n'existent que deux restaurations rapides " Mc Donald's ", montre le décalage entre les visions et les calculs économiques et les réalités locales.

Figure 1 - Variations 2002-2008 des importations et exportations en Nouvelle Calédonie

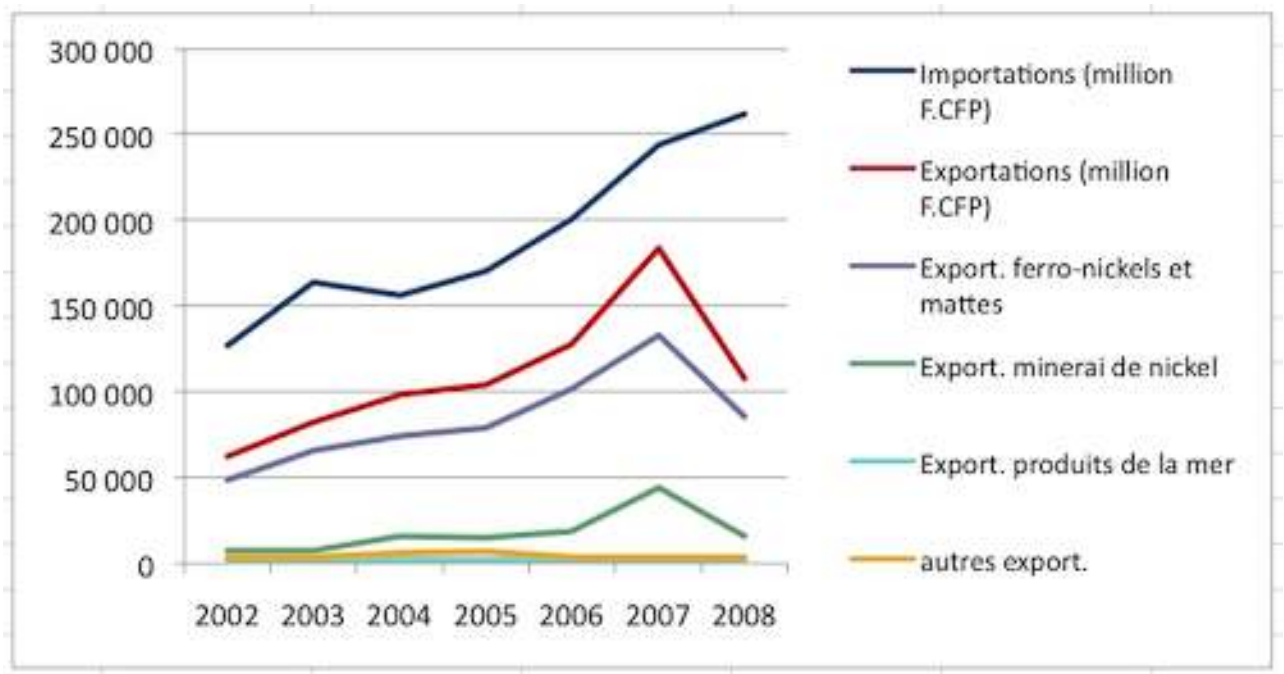

Données : ISEE, 2009

11 Le développement d'un territoire ne dépend pas seulement de celui de l'économie. Il doit être accompagné d'un développement social qui passe par une réduction des inégalités, concept de base de l'aménagement du territoire, mais habituellement affiché dans les discours, mais pas dans la réalité qui privilégie toujours les régions et secteurs performants, sources de richesses du «pays». La prise de conscience de l'existence d'inégalités au sein de la société calédonienne et de l'apparition d'une certaine précarité est récente. Les statistiques fiscales montrent qu'un foyer calédonien sur quatre vivrait en dessous du seuil de pauvreté relative, soit 60000 personnes ou $25 \%$ de la population contre $6,5 \%$ en métropole. Ces chiffres de l'ISEE sont cependant à tempérer, car ils n'intègrent pas les ressources non monétaires de l'économie informelle de brousse (Fig. 2). Certains chiffres affichant 16,3\% de chômeurs en 2004 (ISEE, 2008) sont aussi à prendre avec réserve pour les mêmes raisons. Si le chômage montre une tendance générale à la baisse depuis 2002, son taux reste extrêmement sensible au moindre plan de licenciement ou de CDD. Ainsi la fin des travaux de GoroNickel en 2009 amène 1500 demandeurs d'emploi sur le marché du travail, pouvant faire remonter le nombre total à près de 8000, chiffre comparable à la situation de 1998, si le Prego, "Programme de Remobilisation pour l'Emploi après Goro » ne parvient pas à reclasser tous les demandeurs. On notera cependant que le chômage ne touche plus en Nouvelle-Calédonie que les personnes non ou peu qualifiées.

12 A l'alignement des chiffres qui rassurent les économistes et assurent la base de la politique de développement territorial, s'oppose la fluidité, le flou, l'entremêlement des situations, des espaces et des hommes. 
Figure 2 - Population vivant en tribu

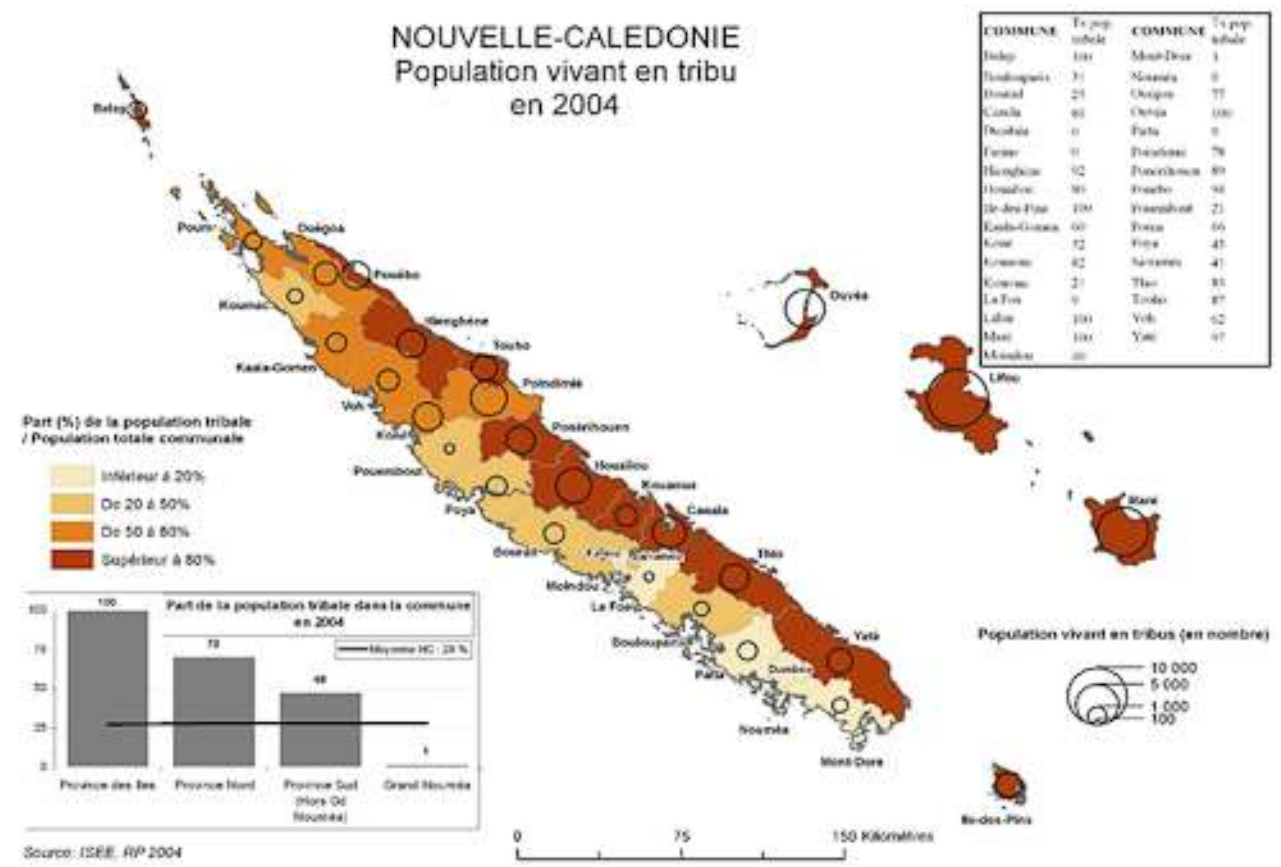

Source : GNC, 2009a. Atlas cartographique. Nouvelle-Calédonie 2025

\section{Terres, espaces et territoire}

Le territoire est caractérisé par trois types d'espaces aux spécificités marquées qui ont des incidences fortes sur les revenus des ménages : l'espace tribal (plus du quart de la population) où l'économie demeure peu monétarisée et la part de l'autoconsommation significative, les villages de brousse et enfin le Grand Nouméa, pôle citadin multiculturel où se concentre la majorité de la population et de l'activité économique. Sur le plan foncier existent aussi trois types de régimes : les terres domaniales, les terres coutumières et les terres privées (Fig. 3). Ceux-ci sont déterminants de l'évolution et de l'aménagement du territoire, mais aussi de la distribution de la population. 


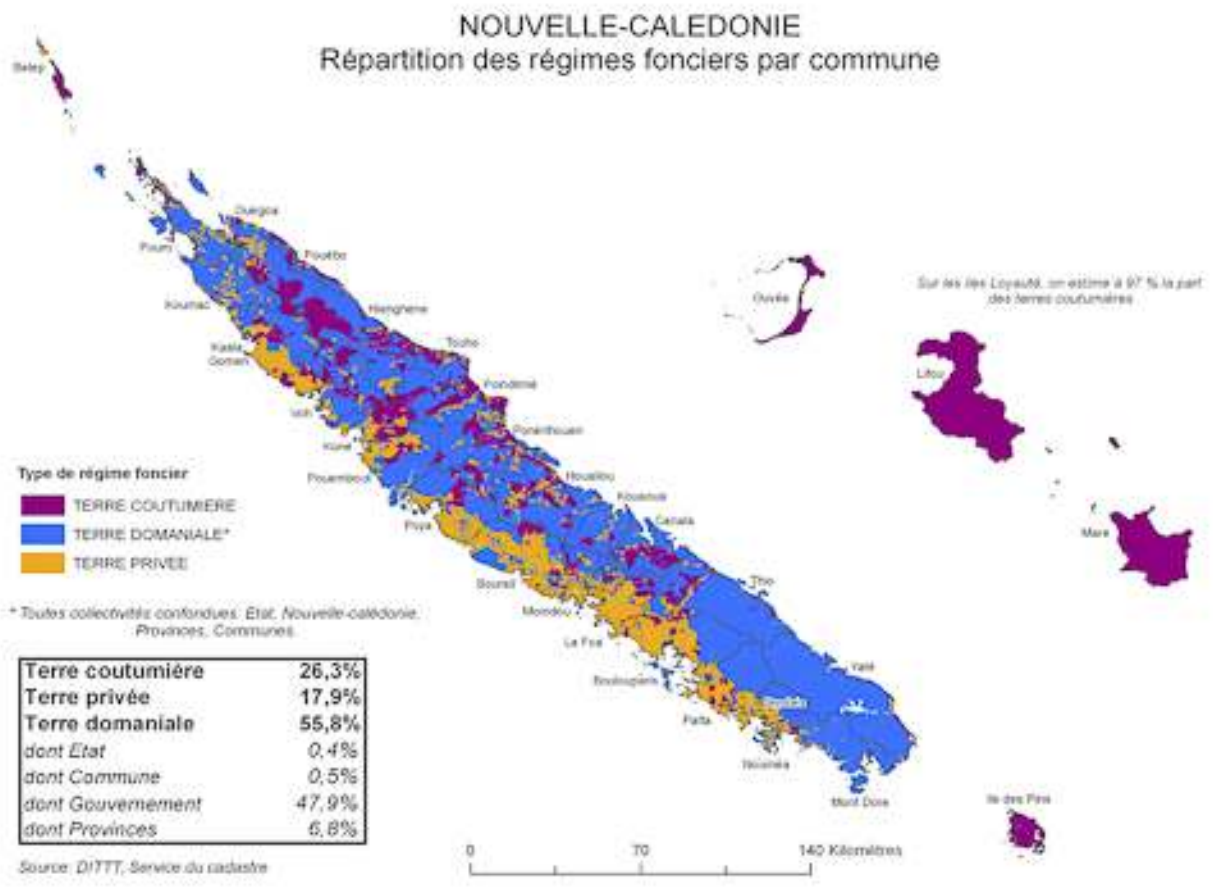

Source : GNC, 2009a. Atlas cartographique. Nouvelle-Calédonie 2025

14 Avec près de la moitié des 245580 habitants (RGP de juillet-août 2009) (ISEE 2009b) concentrée dans le Grand Nouméa, la répartition de la population est une des caractéristiques génératrice des inégalités de développement et de partage des richesses. La recherche d'emploi et le rapprochement des familles alimentent l'exode rural qui perdure, augmentent les installations d'habitat précaire dans des squats, et les actes de délinquance et de violence liés à la vie urbaine et à ses contraintes.

Entre ces espaces, les inégalités de revenus sont fortes. Les $20 \%$ des ménages les plus riches perçoivent $55 \%$ du total des revenus déclarés, contre $40 \%$ en France métropolitaine. Le coefficient de $\mathrm{Gini}^{3}$-qui prend en compte l'ensemble de la répartition des revenus et pas seulement ses extrêmes- confirme ce niveau important d'inégalité monétaire. En 2006, il était de 0,50 pour l'ensemble de la NouvelleCalédonie, similaire à celui de 2000. Plus fortes en Province sud $(0,50)$, les inégalités sont aussi élevées dans les deux autres provinces (Nord, 0,44 ; Iles, 0,41) (CEROM, 2008). Sur le plan politique, alors qu'un nouveau gouvernement a été mis en place en juin 2009 après l'élection à la présidence, le mois précédent, de Philippe Gomes, les mouvements syndicaux de protestation menés par l'USTKE et les blocages de routes et d'aérodromes se répètent et continuent d'alimenter le quotidien des Calédoniens. Ces manifestations $\mathrm{du}$ mécontentement social, qui semble aussi relever du fait français, prennent cependant une autre dimension dans un territoire insulaire où les problèmes sont exacerbés, non pas tant par la cohabitation de deux grandes communautés en présence que sont les Kanak (44\%) et les Européens (34\%) dont les Caldoches, qu'entre un pouvoir politique très libéral et très lié à l'économie du territoire et, la population « de base ".

Rappelons que la Nouvelle-Calédonie, ancien T.O.M., dispose d'institutions spécifiques (Titre XIII de la Constitution) et n'entre pas dans la nouvelle catégorie des collectivités 
d'outre-merétablies par la réforme constitutionnelle de mars 2003. Un statut provisoire a été défini en 1999 en attendant qu'elle se détermine, à partir de 2014, entre l'indépendance et un gouvernement autonome. Elle est donc de ce fait une collectivité suigeneris.

Ce statut provisoire, qui permet le transfert progressif des compétences de l'Etat au territoire (hors compétences régaliennes), et la loi organique de 1999, présentent des imprécisions qui sont source d'importantes difficultés, soulignées par les ateliers de réflexion du SAD sur l'avenir du territoire : «discussions complexes pour savoir qui dispose de quelle compétence normative ; présence de quelques domaines " orphelins"; forte insécurité juridique pesant sur un nombre non négligeable de textes, ...» (GNC, 2009b).

Dans ce contexte d'économie dépendante du cours mondial du nickel, qui héberge en même temps un secteur informel d'autosubsistance et de très petites activités artisanales, l'incertitude politique, en termes de compétences, et les disparités socioculturelles se révèlent comme des contraintes majeures au développement commun " durable ». Les solutions existent pourtant. Elles peuvent se trouver dans une vision globale et une politique prospective originale et audacieuse.

\section{Quelles contraintes d'aménagement et enjeux de développement pour la Nouvelle-Calédonie?}

\section{Des enjeux supra-territoriaux}

Les enjeux de développement que connait la Nouvelle-Calédonie sont multiples. Mais trois d'entre eux se situent au-dessus des autres et doivent être intégrés à chaque pas de la réflexion à mener sur l'avenir du territoire :

1. La réussite d'un destin commun. Ce choix fondamental inscrit dans l'accord de Nouméa écarte a priori les solutions de partage éventuel du territoire en pays distincts. Elle suppose la reconnaissance par tous d'un passé commun, même si divisé, celle de la diversité et même d'identités différentes, avant de pouvoir entrevoir une hypothétique identité commune qui prendra plusieurs générations. La reconnaissance des différentes communautés vivant en Nouvelle-Calédonie, l'affirmation des richesses culturelles de ces communautés, le développement de politiques interculturelles en sont des points importants, enfin affichés comme objectifs dans les réflexions actuelles sur l'avenir. Mais elles doivent déboucher sur des projets concrets et sur une politique générale qui restent à bâtir. Un « destin commun » ne peut se construire sans une égalité de chances, sans la possibilité, paradoxale en apparence seulement, de respecter la diversité et la valeur de ses richesses, véritables atouts pour la communauté globale.

2. Le "rééquilibrage" du territoire, sous-entend une réduction des inégalités de développement, notamment entre le Nord, le Sud et les îles, un rééquilibrage géographique, que l'aménagement du territoire doit et peut résoudre et, un rééquilibrage social qui doit permettre une véritable égalité des chances. Il passe par l'amélioration de l'accès à l'éducation en Province Nord, par la mise en place de l'usine de nickel du Nord, le développement du nouveau pôle urbain VKP autour de l'usine du Nord, par l'aide aux très petites entreprises locales, par le soutien au développement d'activités génératrices de revenus, par une structure d'économie solidaire, par le développement du secteur sanitaire et social, par la réalisation d'études économiques prospectives, et donc, par l'élaboration et la mise en place du Schéma d'Aménagement et de Développement de la Nouvelle-Calédonie (SAD). 
3. Un développement territorial durable ne doit pas se limiter aux principes de base d'une bonne gestion de l'environnement pour soutenir le développement économique, mais doit s'attacher à être aussi un développement social durable et solidaire. Même si la promotion touristique tente de vendre une "île de l'éternel printemps", image paradisiaque où règnent douceur de vivre et absence de contraintes, l'archipel calédonien recense un grand nombre de contraintes naturelles (insularité, endémicité à protéger, relief montagneux barrière, secteurs enclavés, excès climatiques dont cyclones, inondations et sécheresses, fragilité et épaisseur des sols, etc.), auxquelles s'ajoutent les effets directs et indirects de la pression anthropique sur le milieu (mines, urbanisation, pollutions et, conflits communautaires...), le problème majeur restant l'absence d'une politique environnementale globale à l'échelle du territoire déjà déplorée par le passé (Jost, 1996, 1998). Enfin, un développement territorial durable passe nécessairement par une coopération intra et interterritoriale entre les différents territoires et espaces de vie.

\section{Des enjeux territoriaux multiples}

Hormis ces enjeux supra-territoriaux, la Nouvelle-Calédonie doit faire face à d'autres défis pour écrire dans l'histoire un avenir durable. La loi organique a donné les grandes orientations pour le développement et l'aménagement du territoire appuyé sur un schéma, le SAD. La conférence des acteurs réunie le 14 mai 2008 en a lancé les travaux préparatoires suivant une approche en trois temps :

1. La réalisation d'un diagnostic de la Nouvelle-Calédonie soulignant les atouts et les faiblesses, et mettant en avant les grands enjeux auxquels il faut répondre (2008)

2. La formulation d'orientations fondamentales concernant les sujets visés par l'article 211 de l'Accord de Nouméa, à un horizon de 15 ans (2009)

3. L'analyse des moyens à mettre en œuvre par l'Etat, la Nouvelle-Calédonie, les provinces et les communes (fin 2009, début 2010), pour une mise en œuvre au courant du deuxième semestre 2010.

La première phase a été clôturée à la mi-décembre 2009 avec l'adoption par le comité de pilotage du document "Diagnostic et enjeux». Mais en raison des contraintes de calendrier liées à l'échéance du contrat de développement 2006-2010, les $2^{\mathrm{e}}$ et la $3^{\mathrm{e}}$ phases, en démarrage, ont été fusionnées en « orientations/moyens ».

Neuf thèmes ont été retenus pour les neuf ateliers du diagnostic (GNC, 2009b) : 1 : Solidarité sociale et égalité des chances ; 2 : Adéquation population - emploi ; $3:$ Vie et performance des entreprises ; 4 : Mondialisation ; 5 : Développement, culture et valeurs identitaires ; 6 : Environnement et cadre de vie ; 7 : Organisation spatiale - Services à la population et activités; 8 : Organisation spatiale - Occupation du sol, ruralité et urbanisation ; 9 : Administration.

Le bilan, réalisé selon une approche participative, est sans grande complaisance, bien qu'apparaissant parfois quelque peu édulcoré, notamment sur les questions interculturelles. Il s'autosatisfait également des progrès en matière de développement (éducation, santé...). Nous ne reprendrons ici que les conclusions et enjeux qui nous apparaissent les plus importants et que nous complétons par notre analyse de la situation. 


\section{Des enjeux socioculturels déterminants} ate que «les modèles de développement traditionnel et occid toujours présentés comme étant en totale contradiction, ... [alors qu'] ils sont plutôt à regarder comme complémentaires. D'une part, les Kanak souhaitent, pour beaucoup, pouvoir s'intégrer à une économie de marché, qu'ils savent indispensable à la construction du pays. D'autre part, tradition et modernité n'ont rien d'incompatibles. Des passerelles existent, d'autres sont à inventer en particulier grâce à l'adaptation des réglementations, de la fiscalité, du code du travail. Il s'agit de permettre l'émergence de formes différenciées de développement (autosubsistance, économie mixte, pluriactivité, production marchande...) et leur coexistence au sein d'un même espace socio-économique. Les politiques culturelles provinciales répondent aux attentes spécifiques de chaque type de population. On dénonce cependant une absence de vision d'ensemble pour une meilleure lisibilité des actions à l'intérieur comme à l'extérieur du territoire » (GNC, 2009b).

Ce constat d'une absence de vision, sans grande compromission, souligne l'absence de politique générale. Tant que les politiques et décideurs eux-mêmes ne seront pas convaincus d'une communauté de destin et dès lors capables de projeter toute la population dans l'avenir, il n'y aura pas de vision commune possible du futur. Quels échanges et compréhension des problèmes réciproques peut-il y avoir entre les décideurs Caldoches de la Province sud enfermés dans la "Maison bleue " près des marinas de Nouméa et le petit chef de la tribu de Tchambouene sur la côte nord-est qui réussit à remplacer l'alcool par le kava et à apaiser les tensions locales? Quelle perception de l'avenir peut avoir la population de Goro face au géant minier qui fouille 
ses terres et souille son lagon, après lui avoir promis des emplois, finalement donnés à une main-d'œuvre philippine importée?

L'analyse de la question de la solidarité sociale et de l'égalité des chances peut être faite au regard «de l'éducation, de la santé, du logement, de la politique familiale, de la solidarité et des tarifs sociaux des services au public », comme l'a fait un atelier du SAD en charge de cette question. Le bilan qui en ressort est le suivant : "Le système éducatif calédonien est d'un bon niveau... mais des disparités, potentiellement facteurs d'échec scolaire, existent, liées à l'éloignement géographique et aux difficultés d'intégration culturelle de certains ", "Le système de santé est également satisfaisant... mais la forte concentration des spécialités médicales à Nouméa et les difficultés de déplacement pour certaines populations tempèrent ce constat, de même que les différences entre provinces concernant l'aide médicale : les populations les plus fragiles sont aussi les moins informées et les moins bien couvertes... " (GNC, 2009b).

Auto satisfecit expliquant l'échec, scolaire ou autre, par les «difficultés d'intégration culturelle de certains »? L'analyse ne pose pas la question de la différence de références ou de perceptions culturelles, d'espaces perçus, d'espaces vécus, de l'adéquation des programmes, d'une conception différente du développement et de l'accès à l'égalité... considérant encore "l'intégration » au modèle occidental, comme seule voie possible, alors que l'on reconnaît de plus en plus en Europe les apports de la différence comme source d'enrichissement à valoriser.

Pour permettre une harmonie sociale, l'accès aux services et au logement pour tous est nécessaire. Mais les logements font défaut sur l'agglomération de Nouméa du fait de son attractivité économique et scolaire et déjà sur la zone VKP dynamisée par le projet Koniambo. Les conséquences en sont une sur-occupation des logements et une augmentation de l'habitat précaire en squats. L'absence d'un politique générale du logement et le manque d'outils réglementaires, du fait du partage des compétences, sont mis en avant pour expliquer cet état de situation.

Les transports collectifs, indispensables pour accéder à l'éducation, à l'emploi et à la santé, sont trop onéreux et mal organisés. Sur le Grand Nouméa, seuls $8 \%$ des trajets domicile-travail sont effectués en transports en commun, alors que $77 \%$ sont effectués en voiture (CEROM, 2008). Cette situation explique en soi les nombreux embouteillages et constitue un frein au développement économique. Là aussi, un choix politique de développement d'un réseau de transport efficace à l'échelle du territoire s'impose.

\section{Des contraintes socio-économiques structurelles face à la mondialisation}

En dehors du nickel, du tourisme et de l'aquaculture, l'activité des entreprises calédoniennes est presque entièrement tournée vers le marché intérieur qui est très limité. Avec plus de 95\% des exportations en valeur, le nickel a un effet d'entraînement de l'économie locale qui la rend en même temps très dépendante des fluctuations du marché mondial. Cette dépendance est ressentie comme un risque, mais son impact n'a jamais été mesuré. Les effets de la crise financière mondiale en sont une illustration. Après une année 2007 particulièrement exceptionnelle, le secteur minier et métallurgique a marqué en 2008 un retournement de tendance : les exportations de nickel ont reculé de $17,8 \%$ en volume et chuté de $42,0 \%$ en valeur, lourdement impactées par un cours du nickel qui a perdu presque la moitié de sa valeur (de 16,89 en 2007 à 9,55 US\$/Lb en 2008) (ISEE, 2008). 
Toutefois, le secteur du nickel est amené à se développer avec la mise en œuvre de trois grands projets industriels: les deux programmes de construction d'usines métallurgiques, celle de Goro-Nickel dans le sud et l'usine du nord à Voh (projet Koniambo) et, l'augmentation de $25 \%$ de la capacité de traitement de l'ancienne usine de Doniambo à Nouméa dont la production va s'accroître de 60000 à 75000 tonnes à partir de 2009 (Fig. 4). Leur réalisation devrait permettre à la Nouvelle-Calédonie de rendre excédentaire sa balance commerciale. Au total les créations d'emplois directs devraient approcher les 2000 en phase d'exploitation (2009-2010) et les indirects dépasseraient les 4000 (Les Échos, 10/1/2006). Rappelons que le projet Koniambo est issu d'une volonté politique de rééquilibrage économique et social, initiée en 1988 par les accords de Matignon et confirmée dix ans plus tard par celui de Nouméa. Voh, Koné et Pouembout (VKP), les trois communes concernées par le projet, devraient voir leur population doubler et passer à 16000 habitants d'ici 2015, dans une province qui n'en compte actuellement que 45000 dont les trois quarts vivent en tribu.

Figure 4 - L'activité du nickel en 2008 : centre miniers en activité et usines

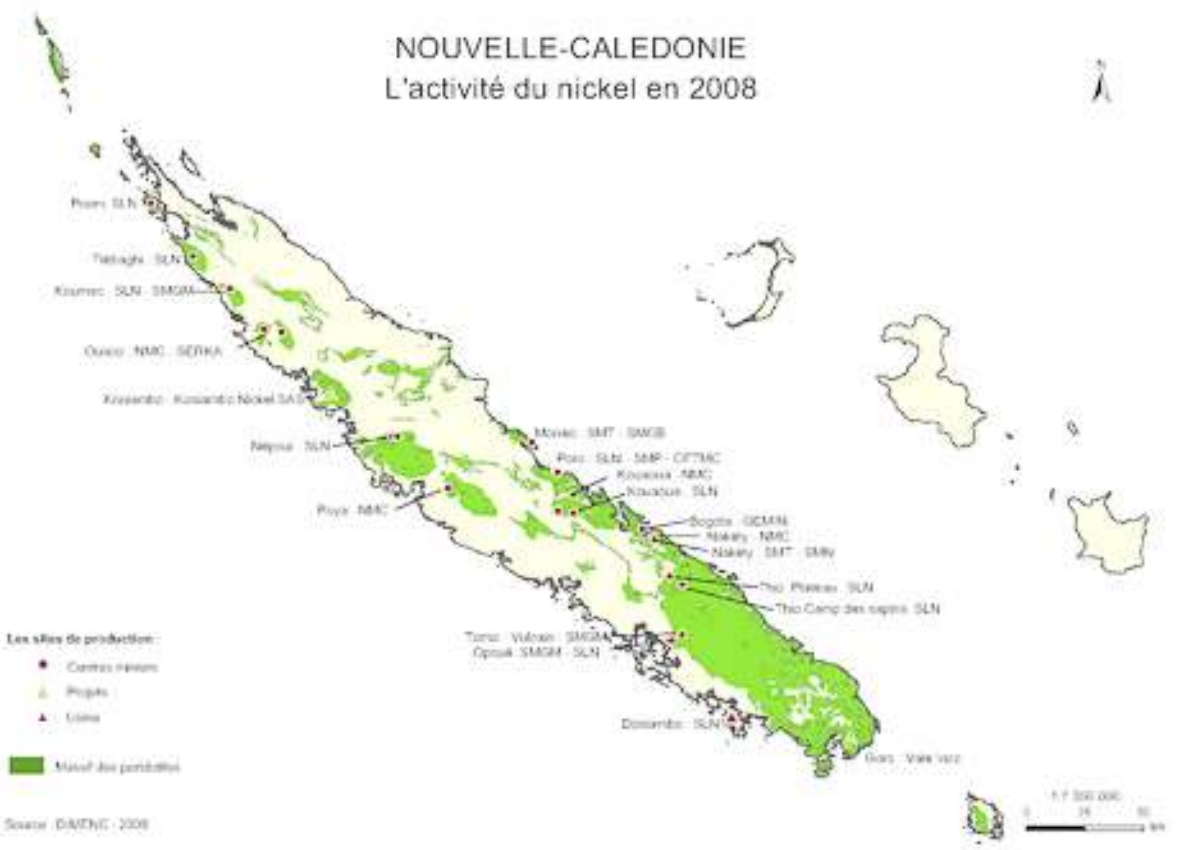

Source : GNC, 2009a. Atlas cartographique. Nouvelle-Calédonie 2025 

l'Australie en septembre 2008 par un câble en fibre optique, sont un moteur indispensable pour les entreprises et les investissements. Mais pour le moment ils semblent seulement, selon l'analyse qui en est faite localement, "multiplier les ouvertures sur le monde, entraîner une augmentation des voyages [!], une consommation plus diversifiée » et, " pour certaines communautés, un décalage qui peut renforcer le repli identitaire et la crainte d'un afflux d'immigrants, le solde migratoire annuel n'étant pourtant que d'environ 1000 personnes»(GNC, 2009b). Cette frilosité à l'égard de nouveaux migrants qui peuvent prendre des emplois aux « Locaux», est ancienne et rédhibitoire, tant au sein des communautés locales qu'au niveau des politiques qui ont fait inscrire dans l'Accord de Nouméa une durée de résidence sur le territoire de dix ans en 1998 et de vingt ans en 2013 pour pouvoir participer aux élections provinciales et territoriales.

(titude générale peu amène à l'égard de l'étranger au territoire, le niveau élevé des prix, la quasi inexistence de transports en commun routiers, le coût et le monopole des transports aériens, expliquent en grande partie que le tourisme, considéré pourtant comme vecteur potentiel de développement, ne parvienne pas à attirer plus de 100000 visiteurs par an et ne représente ainsi que $2,7 \%$ du PIB et $6 \%$ de la population active. La mise en œuvre d'un "Plan de développement touristique concerté" est la solution envisagée, mais quel contenant et quels effets aura-t-il ? Prévoit-il la maîtrise de l'anglais pour tous et du japonais pour accueillir les voisins?

D'autres contraintes limitent le développement du territoire et doivent trouver réponse face aux enjeux de la mondialisation. Ils ont été clairement identifiés par le diagnostic : «la productivité insuffisante de la main-d'cuvre, les formations peu ouvertes à l'international, les barrières douanières en place, l'absence de système de normes dont pourraient se prévaloir les entreprises, le Franc Pacifique (XPF) à portée uniquement régionale, limité à 500000 utilisateurs et, bien sûr, le coût des importations, du fait de l'éloignement et de l'insularité » (GNC, 2009b).

fois de plus est déploré le manque de réflexion stratégique, nécessaire pour mieux identifier les secteurs économiques qui doivent être soutenus à l'export et, pour trouver l'équilibre entre les produits à importer et ceux qui peuvent être produits localement. La Nouvelle-Calédonie est en fait peu attractive pour les investisseurs extérieurs, qui pour le moment ne s'implantent localement que dans l'objectif d'accompagner les projets miniers.

\section{Des contraintes d'aménagement et des enjeux environnementaux indissociables}

L'environnement de la Nouvelle-Calédonie est emblématique d'une biodiversité et d'une endémicité préservées exceptionnelles, dont la population tire une partie de son identité et de sa fierté au plan international, mais dont les ressources sont encore à explorer. Endémique à $80 \%$ la flore néo-calédonienne est en inventaire, mais certaines espèces, comme les plantes concentratrices de nickel ou recolonisatrices de sites miniers stériles, ne sont pas les moins intéressantes (Fig. 5). Les recherches en termes de potentiel pharmacologique ont longue vie devant elles. La biodiversité, surtout animale, est cependant bien plus grande dans le milieu marin et, notamment lagonaire, que dans le milieu terrestre. 
Figure 5 - Comparaison du nombre d'espèces endémiques

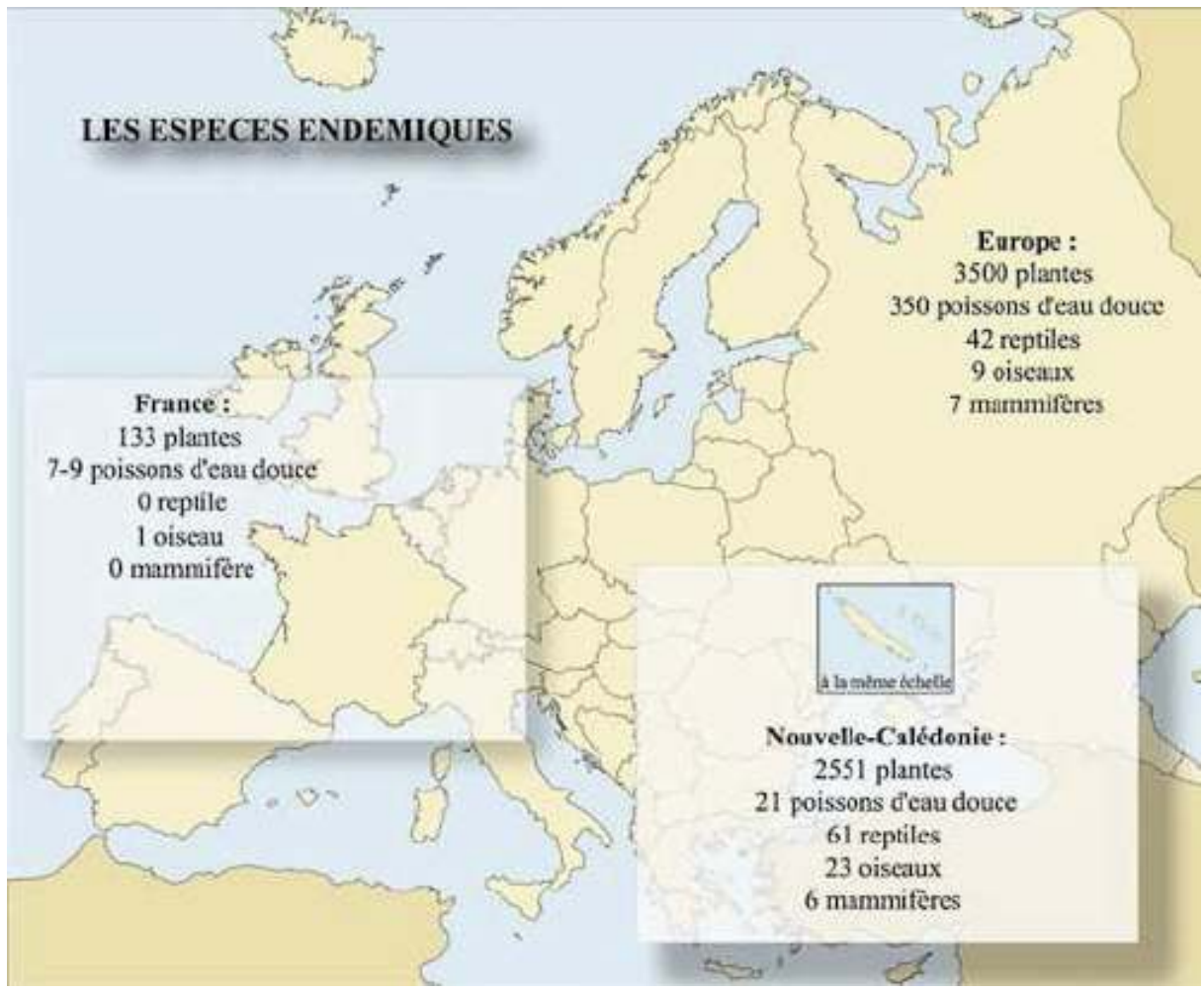

Source : Rapport final de l'atelier $n^{\circ} 6$ « Environnement et cadre de vie », SAD. Données UICN

La reconnaissance partielle de cette richesse est venue de l'UNESCO qui a inscrit en 2008 une partie significative des récifs coralliens et les écosystèmes associés de la Nouvelle Calédonie au patrimoine mondial, premier site d'Outre-Mer à bénéficier de cette inscription. Il fut pourtant en Nouvelle-Calédonie l'objet d'un débat animé entre opposants au classement qui y voyaient une source d'afflux de touristes supplémentaires, ingérables et destructeurs par effet de nombre, et les favorables au classement qui y voyaient une opportunité d'obtenir des moyens supplémentaires de protection et donc de meilleure gestion. L'avenir dira ce qu'il en est, mais il est vrai que le récif calédonien est l'un des rares à être en relatif bon état (site http:// www.reefbase.org/main.aspx)

Soulignant les efforts et les progrès accomplis, le diagnostic de l'atelier «Environnement et cadre de vie » du SAD, présidé par Yves Magnier, ancien membre du gouvernement, fait un constat sans concession : « Le droit de l'environnement est encore peu développé, et la conformité au droit international insuffisante ... Au regard de la fragilité et de la valeur patrimoniale de l'environnement, les moyens alloués sont faibles en matière de gestion des déchets, de gestion de l'eau et d'assainissement, de protection de la biodiversité, de maitrise de la demande en énergie et d'énergies renouvelables... Les outils manquent également en matière d'analyse, de surveillance et de diagnostic environnemental... Le taux de dépendance énergétique du pays est de $96,5 \%$, et sera aggravé par la mise en service des deux nouvelles usines métallurgiques [Goro-Nickel et l'usine du Nord] et l'augmentation de la capacité de production de l'usine de Doniambo. En l'absence de politique de compensation, le niveau des émissions de gaz à effet de serre devrait significativement dépasser les 8 millions de tonnes équivalent $\mathrm{CO}$, ce qui est considérable en regard de la taille et de la population du pays... » (GNC, 2009b). 
à l'avenir réservé à l'environnement. Les principes de base du développement durable sont insuffisants ici, ou vainement édictés pour le moment : l'objectif d'une population stable à terme est encore loin d'être atteint avec un taux d'accroissement naturel de $1,15 \%$ en 2007 (contre $0,43 \%$ en métropole) et une projection d'au moins 289500 habitants en 2030 (Broustet, 2009) ; le principe de l'augmentation de la productivité plutôt que la multiplication des unités de production, est balayé par la construction de deux nouvelles usines de traitement du nickel, dont l'une à l'acide sulfurique, et à la montée en puissance d'une troisième; celui du remplacement des ressources non renouvelables par des ressources renouvelables se limite à quelques dizaines d'hectares reboisés sur sites miniers; enfin, celui d'une exploitation des ressources renouvelables dans la limite de leur capacité de régénération est bafoué par le pompage excessif de l'eau de creeks et rivières, le labourage au bulldozer, les incendies volontaires de forêt, etc.

Parler de développement et d'avenir de la Nouvelle-Calédonie doit revenir dans ce contexte à parler de développement raisonné et raisonnable, intercommunautaire et, surtout, de développement éthique et solidaire, qui serait alors un véritable développement soutenu, durable. L'enjeu de bonne gouvernance est donc de taille, vu la proximité du milieu économique et du milieu politique. Elle est indissociable de l'application des principes de développement durable et d'un développement éthique et solidaire, associé à l'efficacité économique, à l'équité sociale et à la préservation de l'environnement. A l'instar d'un développement économique qui ne prendrait pas en compte l'environnement et ne serait donc pas durable, l'absence d'éthique dans la gouvernance finit par être contreproductive de développement (Dherse, 1998). Il n'est que de voir la crise des "subprimes", de l'économie virtuelle et leurs conséquences pour s'en convaincre. L'application de ce principe, défi majeur à relever, concerne tous les gouvernements de la planète, mais devient vital dans les micro-états et territoires insulaires à faible population et extension, qui connaissent trop souvent alternance de pouvoirs et conflits d'intérêt avec la sphère économique. La Nouvelle-Calédonie n'est pas épargnée et, si l'histoire a déjà montré les imbrications entre pouvoir politique et économie locale, l'avenir n'en sera pas exempt, si une réforme des institutions n'est pas entreprise. C'est là un fait patent, qui a été plusieurs fois mentionné dans les ateliers de préparation du SAD comme un problème urgent auquel s'attaquer. Mais ceci ne peut

EchoGéo, 11 | 2010 
n'être résolu que par une législation renforcée et adaptée, une applicabilité, une application des textes de lois existants et une clarification des compétences et du droit.

Le diagnostic réalisé pour préparer le SAD a fait l'objet d'un travail remarquable qui a associé des centaines de participants (504 exactement) à travers tout le territoire. L'ensemble de la production est très riche en idées et en constats sincères. Ceux-ci restent cependant ourlés de propos conciliateurs qui révèlent l'étroitesse de la marge de manœuvre dont dispose la marche intercommunautaire vers le futur. La production est importante et le bilan apparait à la hauteur des attentes. Il montre toutefois une réflexion endogène, bien naturelle, compréhensible et même voulue, mais qui gagnerait à une certaine distanciation, à une ouverture, déjà existante et à saluer, à amplifier en une prospection d'idées novatrices encore plus grande venant de l'extérieur.

\section{Quelques pistes de réflexion}

51 Le SAD devra comporter des procédures pour améliorer la coordination entre les institutions, pour favoriser l'efficacité de l'action publique, et devra prévoir une meilleure évaluation des politiques publiques et doter le territoire d'observatoires, favoriser les débats libres et libérer les médias de l'influence du pouvoir local.

Il s'agira aussi d'assister les politiques dans leurs décisions, de fournir aux décideurs des outils d'aide à la décision de type tableau de bord, systèmes d'alerte et d'indicateurs, systèmes de support de la décision (SSD). Les collectivités locales et les institutions provinciales se sont dotées de SIG et de bases de données qui ont fait l'objet d'un développement considérable ces dernières années. Il n'y a qu'un pas pour les développer encore en SSD.

Sur le plan de l'urbanisation, l'enjeu du Grand Nouméa (qui n'a pas encore d'existence officielle) n'est pas dans ses rôles de port d'entrée dynamisant le reste du territoire, ou celui de pôle de rayonnement vers l'extérieur, qui ne faibliront pas. Il est plutôt dans celui de modérateur de sa vocation territoriale au profit d'un rééquilibrage vers la zone urbaine émergente de VKP (Voh, Koné, Pouembout) et vers les îles, si isolées. Pour ce faire, l'autorité territoriale qu'est le gouvernement pourrait envisager la mise en place de mesures incitatives au départ, voire contraignantes (taxation, fiscalité,...), pour favoriser une décentralisation de certaines fonctions et activités. L'urbanisation discontinue qu'a connue la commune de Nouméa dans les années 1970-1980, se reproduit aujourd'hui dans sa périphérie. Les effets de coupures et de coutures urbaines se multiplient et renforcent les risques de conflits et d'exclusion. D'autres enjeux se dégagent de l'urbanisation rapide du Grand Nouméa, tel celui, vital pour la respiration de la ville, de la fluidification des transports croissants qu'engendrent les migrations pendulaires, tel celui de la résorption des zones d'habitats précaires, ou celui de trouver des vocations aux terres coutumières que la zone comporte et qui favorisent cette urbanisation en taches discontinues (Fig. 6).

Pour ces dernières, malgré, ou, du fait des imprécisions du statut particulier de la propriété, quelques pistes de réflexion peuvent être avancées: développer une agriculture urbaine, à peine émergente, une horticulture d'approvisionnement de la ville qui trouverait sans doute écho auprès des tribus en charge de ces terres; une autre peut être celle d'une mise en valeur sous forme de zones de loisirs et d'échanges interculturels adaptés et acceptables par toutes les communautés ; une autre encore, de 
zones vertes et/ou de village éco-culturel avec chemins coutumiers acceptant visiteurs contre droits d'entrée, ou la combinaison de plusieurs formes d'usages à définir par études et enquêtes appropriées.

Figure 6 - Répartition du type de terres dans l'agglomération du Grand Nouméa

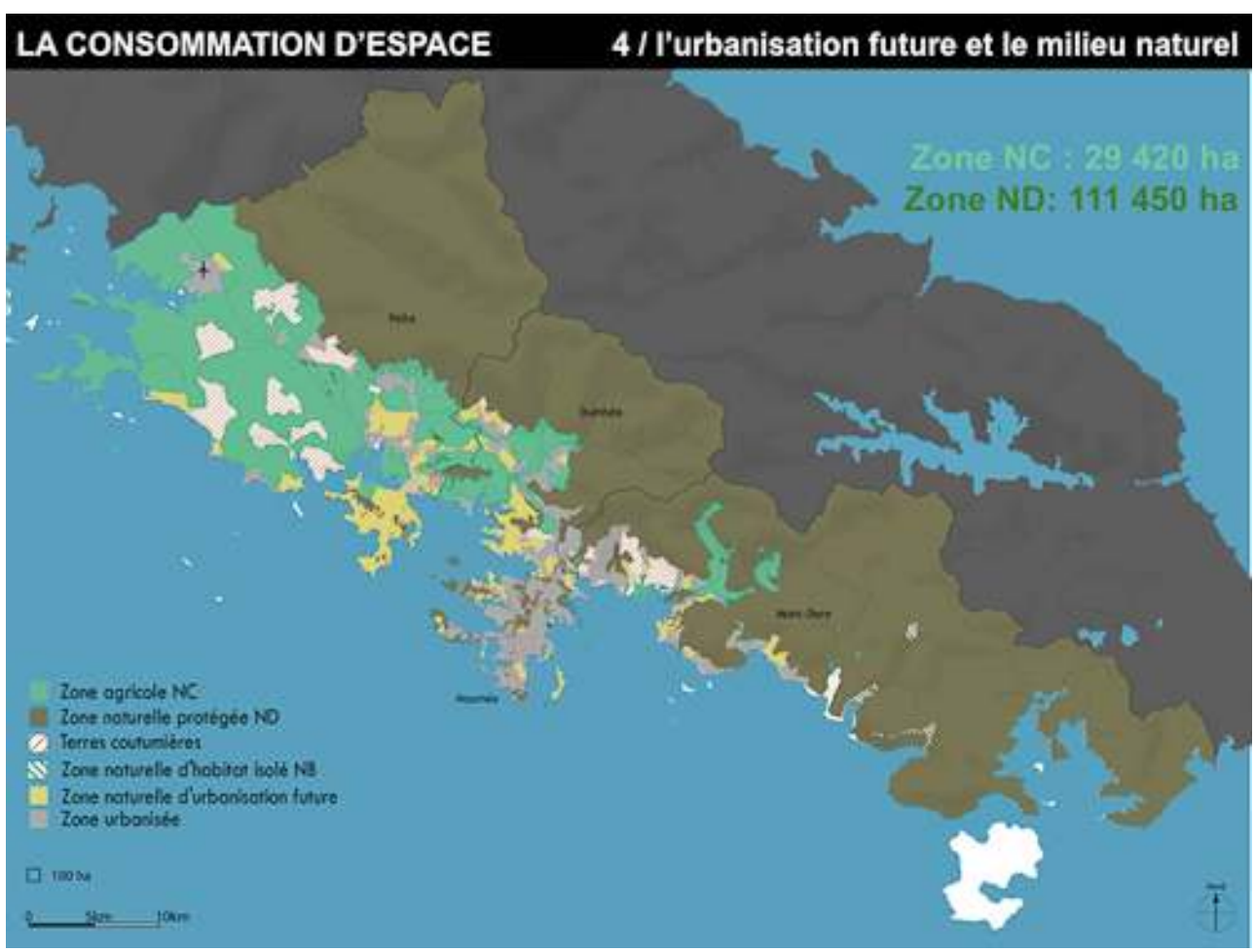

Source : SCAN, 2008

D’autres pistes de développement peuvent être avancées.

- - Diversifier et étendre l'économie calédonienne au-delà de la seule exploitation du nickel, dont le développement des projets miniers doit augmenter les recettes publiques locales et permettre, à travers notamment la mise en place d'une politique fiscale adaptée, cette nécessaire diversification.

- - Réfléchir à ce que n'ont pas développé les grands voisins australien et néo-zélandais. Quelles sont les spécificités du milieu naturel et du milieu humain néo-calédoniens, enrichies du savoir-être et du savoir-faire des différentes communautés, qui apporteraient réponse ou nouveauté sur le marché régional? En dépit de l'influence dominante de l'Australie sur la Mélanésie anglophone, pourquoi ne pas se tourner vers les pays frères de la Mélanésie dont l'immense Papouasie-Nouvelle-Guinée, avec ses 5,2 millions d'habitants, qui est en marche vers une transition économique et socioculturelle génératrice de besoins et de savoir-faire?

- - Développer toutes les formations pour permettre à la population d'atteindre rapidement un bilinguisme français-anglais de façon à mieux s'inscrire dans la région et commercer avec les voisins, tous anglophones, apparaît comme un impératif de simple bon sens. Le renforcement de la formation au japonais apparaît tout aussi important pour le développement économique et touristique du pays et pour améliorer l'image d'accueil toujours si mauvaise à ce jour.

- - Augmenter les capacités de recherche/développement dans les biotechnologies, en s'appuyant sur l'atout exceptionnel que représentent la diversité et l'endémicité des plantes 
de Nouvelle-Calédonie est un axe très porteur de développement. Les biotechnologies jouent un rôle de plus en plus important dans le secteur des industries de santé, mais ont aussi un rôle émergent dans les secteurs de l'environnement, de l'agriculture, de l'agro-alimentaire, ainsi que pour la mise au point de processus industriels innovants. Pourquoi ne pas imaginer l'implantation ou la création d'unités de transformation et de production en s'appuyant sur les travaux de l'IRD et de l'Institut Pasteur présents sur le territoire?

- - Le potentiel pharmaceutique, si peu connu, des plantes endémiques fait l'objet de recherches depuis de nombreuses années. Mais la valorisation et la commercialisation des produits issus de la recherche nécessitent un renforcement de partenariats, une compétence et une direction de ce secteur d'avenir qui soient territoriales.

- - Développer les filières de l'aquaculture et notamment celle de la culture des algues et des micro-algues semble pouvoir être une voie royale pour demain. Nouvel or vert, alternative énergétique au pétrole, capable de produire de l'énergie sous formes d'hydrogène, de biocarburant ou encore de biogaz, non compté le potentiel alimentaire pour l'élevage des mollusques ou l'aquaculture de poissons, le potentiel des algues de tous types est incommensurable et encore largement méconnu. La présence d'un centre IFREMER en est déjà un élément porteur.

- - A l'instar des pays qui ont suivi dès 1989 les recommandations du rapport Brundtland, un Plan d'action environnementale (PAE) pourrait être mis en place et répondre aux carences tant institutionnelles, législatives, que d'évaluation d'impacts des activités humaines. L'association d'experts locaux et d'experts indépendants et extérieurs au territoire apporterait complémentarité et garantie d'une évaluation et de propositions indépendantes.

- - On peut retenir l'idée d'une « structure plus restreinte de recherche-action » qui, à côté « de l'IAC et de l'IRD, permettrait aux institutions, par ses enquêtes, tout à la fois de mieux appréhender les dynamiques collectives du pays et d'alimenter en informations fiables l'indispensable débat public » (Godin, 2009). A l'échelle territoriale cette structure pourrait effectivement être source d'une réflexion/action extrêmement utile sous réserve que lui soit accordé crédit d'une autonomie de réflexion et qu'elle puisse avoir distanciation suffisante pour ne pas être sous obédience.

La liste des possibilités et des choix à faire est longue ... mais, sans utopie le monde n'est plus humain, osons!

\section{Conclusion}

L'avenir de la Nouvelle-Calédonie semble désormais bien pris en main par tous les Néocalédoniens. Un état des lieux de grande portée a été réalisé et constitue aujourd'hui un socle sur lequel bâtir des propositions d'avenir, définir des orientations stratégiques de développement, construire une politique générale, qui a tant fait défaut jusque là. C'est le constat principal qui apparaît de manière récurrente dans tous les secteurs investigués. Ce diagnostic aura surtout eu le formidable effet de faire réfléchir et travailler ensemble toutes les communautés, toutes les parties sur un projet d'avenir commun. C'est bien d'une démarche participative et prospective dont avait besoin le territoire, pour se projeter dans l'avenir sur une voie de destinée commune. Certes, ce bilan montre de nombreuses faiblesses $\mathrm{du}$ fonctionnement actuel $\mathrm{du}$ monde calédonien ; il révèle les dysfonctionnements, les enjeux, et indirectement les risques à ne pas répondre aux défis identifiés. Mais il a été réalisé dans ce but et ne cherche pas d'excuses. Il présente surtout le grand avantage de présenter pour la première fois un 
"système » commun de fonctionnement à l'échelle du territoire, un véritable anthroposystème calédonien, au sens d'entité structurelle et fonctionnelle qui prend en compte les interactions sociétés-milieux et intègre sur un même espace un ou des soussystèmes naturels et un ou des sous-systèmes sociaux, l'ensemble co-évoluant dans la longue durée et comportant plusieurs échelles spatio-temporelles d'analyse (Levêque, 2003). Il rejoint par là le concept de géosystème (Jost, 1996), en mettant l'accent sur le rôle déterminant joué par les sociétés humaines, ici, les communautés calédoniennes en présence. C'est dans cette voie que la Nouvelle-Calédonie trouvera les fondements de sa construction de pays, voire un jour, de nation. C'est par la mise en évidence et le développement d'un mode ou d'un système de fonctionnement commun, ou plutôt coopératif, de sous-systèmes combinant écosystèmes et anthroposystèmes, que chaque entité pourra s'épanouir et se valoriser en travaillant avec l'autre. C'est pourquoi, sur le plan géographique et politique, pourrait être repensé le découpage territorial en fonction de ces entités, en distinguant les différentes combinaisons d'espaces que sont, dans l'ordre, les différentes aires culturelles, puis les unités naturelles, les zones de potentiel économique, puis seulement les territoires administratifs. La définition de nouveaux anthroposystèmes et/ou géosystèmes permettrait de mettre en évidence des petits « pays » à une échelle intermédiaire entre les communes et les provinces. Le SAD pourra, voire devra, reconnaître ces pays ${ }^{4}$, au sens de région naturelle correspondant à une aire culturelle (ou pays traditionnel).

Le SAD est l'occasion unique pour la Nouvelle-Calédonie de devenir un Pays, sans connotation indépendantiste, au sens "Territoire de projet» caractérisé par une cohésion géographique, économique, culturelle ou sociale ${ }^{5}$, et ce, au-delà du simple affichage actuel qui relève plus de la méthode Coué que de la réalité.

Les Néo-calédoniens viennent de poser, seuls et unis, la première base fondatrice de leur maison commune. L'étape suivante est la projection architecturale de l'édifice qui devra pouvoir évoluer et résister au temps.

Tocqueville, De la démocratie en Amérique :

«C'est en chargeant les citoyens de l'administration des petites affaires, bien plus qu'en leur livrant le gouvernement des grandes, qu'on les intéresse au bien public et qu'on leur fait voir les besoins qu'ils ont sans cesse les uns des autres pour le produire.

On peut, par une action d'éclat, captiver tout à coup la faveur d'un peuple ; mais, pour gagner l'amour et le respect de la population qui vous entoure, il faut une longue succession de petits services rendus, de bons offices obscurs, une habitude constante de bienveillance et une réputation bien établie de désintéressement ».

\section{BIBLIOGRAPHIE}

Bouard S., Sourisseau J.-M. et Pestana G., 2008. La participation des acteurs locaux à l'élaboration et l'évaluation des politiques publiques de développement durable en Nouvelle-Calédonie. EchoGéo, n7, 2008, http://echogeo.revues.org/index9693.html 
Broustet D., 2009. Projections de population pour la Nouvelle-Calédonie à l'horizon 2030 «Une évolution entre croissance et vieillissement ». Rapport ISEE Nouvelle-Calédonie, $6 \mathrm{p}$.

CEROM, 2008. Les défis de la croissance calédonienne. Nouméa, Comptes Economiques Rapides de l'Outre-Mer/ISEE, décembre 2008, 60 p.

DAVAR-ISEE, Recensement Général Agricole 2002/Inventaire communal. Notes et Documents $\mathrm{n}^{\circ} 91$, 92 et 93, novembre 2004. Disponible en ligne sur : www.isee.nc

Dherse J.L. et Dom H. Minguet, 1998. L'éthique ou le chaos. Paris, Presses de la Renaissance, 382 p.

Godin P., 2009. Enquête sur les petites entreprises kanak en tribu. Synthèse des entretiens et éléments d'analyse. Rapport Nouvelle-Calédonie 2025. Schéma d'Aménagement et de Développement de la Nouvelle-Calédonie. Nouméa, Gouvernement de la Nouvelle-Calédonie/ Haut-commissariat de la République en Nouvelle-Calédonie, SAD, mai 2009, 23 p.

Gouvernement de la Nouvelle-Calédonie (GNC), 2007. Rapport de la Nouvelle-Calédonie sur le développement infrastructurel. Nouméa, Commission économique et sociale pour l'Asie et le Pacifique, juin 2007, $104 \mathrm{p}$.

Gouvernement de la Nouvelle-Calédonie (GNC), 2009b. Rapports des 9 ateliers du diagnostic. Nouvelle-Calédonie 2025. Schéma d'Aménagement et de Développement de la Nouvelle-Calédonie. Nouméa, Gouvernement de la Nouvelle-Calédonie/Haut-commissariat de la République en NouvelleCalédonie, SAD, janvier 2009, 309 p.

Gouvernement de la Nouvelle-Calédonie (GNC), 2009a. Atlas cartographique. Nouvelle-Calédonie 2025. Schéma d'Aménagement et de Développement de la Nouvelle-Calédonie. Nouméa, Gouvernement de la Nouvelle-Calédonie, $90 \mathrm{p}$.

IEOM, 2008. La Nouvelle-Calédonie en 2007. Rapport annuel de l'IEOM. Paris, IEOM, 237 p.

ISEE, 2009b. Recensement de la population. http://www.isee.nc/recenspop/rp2009.html

ISEE, 2009a. Chiffres-clés de la Nouvelle-Calédonie. Nouméa, ISEE, 29 mai 2009, 2 p.

ISEE, 2008. Bilan économique et social 2008. Nouméa, ISEE, 58 p.

Jost C., 1996, Contraintes et enjeux environnementaux des territoires français du Pacifique. In Jost C., Le Bourdiec P. et Angleviel F. (eds), Géo-Pacifique - des espaces français, 2e édit., UFP-CTRDP, p. 21-34.

Jost C., 1997, Géosystèmes littoraux et lagonaires du sud calédonien : Interfaces d'échanges terrestres et marins. (346 Ko) in Boyer Ph. (ed), La Mer, espace, perception et imaginaire dans le Pacifique sud, L'Harmattan, Paris, 1997, p. 241-264.

Jost C., 1998, Environmental constraints and issues in the French Pacific territories. In Jost C. (ed.), The French-Speaking Pacific. Population, Environment and Development Issues. Mount Nebo, Australia, Boombana Publications, p. 63-76.

Jouve D. et Pitoiset A., 2008. La Nouvelle-Calédonie. In L'Année Francophone Internationale, $\mathrm{n}^{\circ} 16$, Québec/Paris, CIDEF-AFI, p. 242-245.

Les Échos, 10/1/2006. Nouvelle-Calédonie : les usines de nickel devraient générer 6000 emplois.

Lévêque Ch., Muxart T., Abbadie L., Weil A. et van der Leeuw S., 2003. "L'anthroposystème : entité structurelle et fonctionnelle des interactions sociétés - milieux". In Lévêque Ch. et van der Leeuw S. (éds), Quelles natures voulons-nous ?, Elsevier, Paris, p. 110-129.

Météo France, 2006. Impacts du réchauffement global en Nouvelle-Calédonie. Nouméa, Rapport Météo France réalisé par Maîtrepierre Luc, 17 p. 
SCAN Nouméa, 2008. Diagnostic - Enjeux SCAN, Nouméa. Schéma de Cohérence de l'Agglomération de Nouméa. DBW, TETRA, ARTIA, VIA COMMEA, Document provisoire, mai 2008, 134 p.

http://www.nouvelle-caledonie.gouv.fr/

http://www.legifrance.gouv.fr/affichTexte.do?cidTexte=JORFTEXT000000393606

http://nouvellecaledonie.info/2009/03/15/metissage-et-destin-commun/

http://www.province-sud.nc/emploi/candidats/accompagnement-a-la-recherche-demploi/plande-remobilisation-pour-lemploi-apres-goro-prego

http://www.reefbase.org/main.aspx

http://www.ville-noumea.nc/vivre/contrat_agglo2006.asp

http://wwz.ifremer.fr/aquaculture/filieres/filiere_algues/la_decouverte_des_algues/

microalgues

http://www.revue-metallurgie.org/articles/metal/pdf/2006/02/vie.pdf

http://www.hypergeo.eu/article.php3?id_article=270

\section{NOTES}

1. CEROM (Comptes Economiques Rapides de l'Outre Mer), associant l'Agence Française de Développement (AFD), l'Institut d'Emission d'Outre-mer (IEOM) et l'Institut de la Statistique et des Etudes Economiques (ISEE).

2. Face à la difficulté d'avoir les mêmes indicateurs pour tous les pays du monde, certains économistes ont proposés une comparaison internationale à partir du prix du Big Mac. Il s'agit d'un produit uniforme vendu dans le monde entier et dont le prix est déterminé par une combinaison d'éléments formant un type de panier diversifié : produits locaux et importés, salaires, services externes, foncier...

3. Ce coefficient (compris entre 0 et 1 ) indique dans quelle mesure la distribution des revenus constatée dans un pays s'écarte d'une répartition parfaitement égalitaire. Plus ce coefficient est élevé, plus les inégalités de revenu sont importantes. Les pays les plus égalitaires comme le Danemark ou la Japon ont un coefficient de 0,25 , les plus inégalitaires comme le Brésil ou l'Afrique du Sud, de 0,60, la France ayant un coefficient de 0,33.

4. Pays vient du latin pagus qui désignait une subdivision territoriale et tribale d'étendue restreinte (de l'ordre de quelques centaines de $\mathrm{km}^{2}$ ), soit une «petite région naturelle (ou pays traditionnel) caractérisée par un héritage historique féodal ou antique», telle que définie par l'école géographique de Vidal de la Blache.

5. Pour le développement de contrats de pays ; sens de la loi du 4 février 1995 (Loi d'Orientation pour l'Aménagement et le Développement du Territoire). 


\section{RÉSUMÉS}

En 2008 a été lancée en Nouvelle-Calédonie une vaste consultation pour élaborer un Schéma d'Aménagement et de Développement du territoire à l'horizon 2025. Si l'économie calédonienne a connu une forte croissance pendant plus d'une décennie, pouvant soutenir les besoins de la population et les investissements, le grand défi d'aujourd'hui réside dans la capacité à construire une communauté de destin basée sur une reconnaissance d'aspirations multiculturelles et sur un projet commun. Lancé dix ans après l'Accord de Nouméa qui le prévoyait, le S.A.D. est cette chance qu'ont les Néo-Calédoniens d'écrire enfin leur avenir ensemble. La réflexion stratégique globale et la mise en place d'une politique générale restent toutefois encore à mettre en place.

In 2008 a vast consultation was launched in New Caledonia aimed at establishing a development plan for the territory, directed at the year 2025. If the Caledonian economy has experienced high growth over the past decade, thereby satisfying population and investment needs, the current challenge resides in the capacity to build the society of the future based on the recognition of multi-cultural aspirations and a shared project. Envisaged by the Accord de Nouméa and launched ten years later, the SAD offers all New Caledonians the opportunity to plan the future together. The over-arching strategic reflection and the creation of the broad politic foundations for the project have yet, however, to be determined.

\section{INDEX}

Keywords : 2025, constraint, development plan, issue, New Caledonia

Mots-clés : 2025, contrainte, enjeu, Nouvelle-Calédonie, schéma de développement

\section{AUTEUR}

\section{CHRISTIAN JOST}

Christian Jost, géographe, est Professeur des universités et directeur du CEGUM - EA 1105, Centre de recherche en géographie de l'université Paul Verlaine -Metz. Il a créé la filière géographie à l'université de la Nouvelle-Calédonie où il était en poste de 1993 à 2006. Christian.jost@univmetz.fr Parmi ses récentes publications sur le Pacifique : Jost C., 2009. Identités floues : de la coutume tribale à l'appropriation multi-culturelle d'espaces urbains dans le Pacifique. In Santos, A.C., Almeida, C. M., Amorim, C. \& Batalha, M.C., "Identidades fora de foco", Rio de Janeiro : de Letra, juillet 2009, 296 p., ISBN 9785-60559-09-1, p.121-136. Jost C., 2009 et annuel depuis 1997. « Vanuatu » et « Wallis-et-Futuna », L'Année Francophone Internationale. CIDEF-AFI, Québec/ Paris. Jost C. and S. Andrefouët, 2007. Long term natural and human perturbations and current status of Clipperton Atoll, a remote island of the Eastern Pacific. Pacific Conservation Biology , Surrey Beatty \& Sons, Chipping Norton, NSW, Australia, 12, 3, p. 207-218. 